\title{
THE DEVELOPMENT OF THE COLLAGEN FIBRE NETWORK IN TISSUE-ENGINEERED CARTILAGE CONSTRUCTS IN VIVO. ENGINEERED CARTILAGE REORGANISES FIBRE NETWORK
}

\author{
H. Paetzold ${ }^{*}$, C. Goepfert ${ }^{2}$, G. Huber ${ }^{1}$, E. Hoenig ${ }^{1}$, R. Pörtner ${ }^{2}$, A. F. Schilling ${ }^{3}$, N. M. Meenen ${ }^{4}$ and M. M. Morlock ${ }^{1}$ \\ ${ }^{1}$ Biomechanics Section, Hamburg University of Technology Hamburg, Germany \\ ${ }^{2}$ Institute of Bioprocess and Biosystems Engineering, Hamburg University of Technology, Hamburg, Germany \\ ${ }^{3}$ Department of Plastic and Hand Surgery, Technische Universität München, Munich, Germany \\ ${ }^{4}$ Department of Pediatric Orthopaedic Surgery, Children's Hospital Hamburg-Altona, Hamburg, Germany
}

\begin{abstract}
For long term durability of tissue-engineered cartilage implanted in vivo, the development of the collagen fibre network orientation is essential as well as the distribution of collagen, since expanded chondrocytes are known to synthesise collagen type I. Typically, these properties differ strongly between native and tissue-engineered cartilage. Nonetheless, the clinical results of a pilot study with implanted tissue-engineered cartilage in pigs were surprisingly good. The purpose of this study was therefore to analyse if the structure and composition of the artificial cartilage tissue changes in the first 52 weeks after implantation. Thus, collagen network orientation and collagen type distribution in tissue-engineered cartilage-carrier-constructs implanted in the knee joints of Göttinger minipigs for 2, 26 or 52 weeks have been further investigated by processing digitised microscopy images of histological sections. The comparison to native cartilage demonstrated that fibre orientation over the cartilage depth has a clear tendency towards native cartilage with increasing time of implantation. After 2 weeks, the collagen fibres of the superficial zone were oriented parallel to the articular surface with little anisotropy present in the middle and deep zones. Overall, fibre orientation and collagen distribution within the implants were less homogenous than in native cartilage tissue. Despite a relatively low number of specimens, the consistent observation of a continuous approximation to native tissue is very promising and suggests that it may not be necessary to engineer the perfect tissue for implantation but rather to provide an intermediate solution to help the body to heal itself.
\end{abstract}

Keywords: Collagen fibre organisation development in vivo; collagen distribution development in vivo; tissueengineered cartilage; cartilage implant; polarisation microscopy; digital image analysis

* Address for correspondence:

Dr Helge Paetzold

Hamburg University of Technology

Biomechanical Section

Denickestrasse 15

Hamburg 21073

Germany

Telephone Number: +491795944141

FAX Number: +4989918356

E-mail: helge.paetzold@gmail.com

\section{Introduction}

Injuries of joint cartilage in patients can result in a significant loss of quality of life and an increase in the susceptibility to early osteoarthritis (Bhosale and Richardson, 2008). Although the development of osteoarthritis in cartilage has been extensively investigated (Agnesi et al., 2008; Bae et al., 2003; Buckwalter et al., 2000; Nagaosa et al., 2002; Setton et al., 1999; van der Esch et al., 2005) there is currently no surgical method available for cartilage injuries that can prevent its early onset (Magnussen et al., 2008). Tissue engineering offers promising new approaches that have the potential to provide such therapies. The aim of creating vital implants for tissue repair is to provide a fully functional replacement of the damaged tissue. This includes the biomechanical, structural and biochemical properties of the cartilage tissue, as well as its integration within the existing native tissue.

Microscopy is a tool commonly used to study tissue samples. Bright field and fluorescence microscopy are normally used to show the biochemical constituents of the cartilage tissue, for example collagen or glycosaminoglycans, while polarised light microscopy allows the organisation of collagen fibres to be observed (Hughes et al., 2005; Rieppo et al., 2008; Rieppo et al., 2009).

Benninghof introduced the classical model for the architecture of the collagen network, consisting of arcades that are formed by fibres that run perpendicular to the subchondral bone in the deep zone and arciformly change direction in the transitional zone to run parallel to the articular surface in the superficial zone (Benninghoff, 1925). These patterns have been confirmed using polarised light microscopy (Hughes et al., 2005; Hyttinen et al., 2009; Julkunen et al., 2009; Rieppo et al., 2009) and $\mu$-MRI (Alhadlaq et al., 2004; Gründer, 2006; Nissi et al., 2006; Seidel et al., 2005; Xia et al., 2003). None of these studies has investigated the development of the fibre orientation within in vivo implanted tissue-engineered cartilage over time. Further, the methods applied have been limited in their ability to quantify the collagen distribution throughout the area of the tissue.

The purpose of this pilot study was to investigate whether tissue-engineered cartilage implants develop in vivo an anisotropic collagen network after their implantation and if such a network resembles the network found in native cartilage. Further, to study these questions, 
a new method was developed to quantify the orientation and distribution of the collagen fibre network in soft tissues.

\section{Materials and Methods}

\section{TE-cartilage constructs}

Histological sections of tissue-engineered and native cartilage were taken from a previous animal experiment described in detail elsewhere (Baumbach et al., 2008; Nagel-Heyer, 2004; Nagel-Heyer et al., 2005; Petersen et al., 2008). Briefly, osteochondral implants were cultured, consisting of a calcium phosphate carrier ( $\varnothing 4.5 \mathrm{~mm} \times 2$ $\mathrm{mm})$, as a bone substitute, with a layer of tissue-engineered cartilage $(\sim 1 \mathrm{~mm})$. These were implanted in the femoral condyles of six Göttinger minipigs, which were sacrificed after 2,26 or 52 weeks.

The tissue-engineered cartilage was cultured from joint biopsies taken from the knees of six minipigs under general anaesthesia. Chondrocytes were isolated by sequential digestion with hyaluronidase and collagenase and expanded (Petersen et al., 2008). After two or three passages, adherent cells were released from the culture flasks by exposure to $0.25 \%$ trypsin/EDTA. $2-4 \times 10^{5}$ expanded chondrocytes were then seeded on the calcium phosphate carriers to improve the adherence of cartilaginous tissue produced later on. Cell-seeded carriers were cultivated for two weeks (Petersen et al., 2008). To prepare the cartilaginous layer of the constructs, expanded chondrocytes were encapsulated in alginate gel and cultivated for two weeks (Yaeger et al., 1997). After 2 weeks of cultivation, the chondrocytes were surrounded by cell-associated matrix. Cells were released from the alginate gel and re-suspended in medium and sedimented $\left(1 \times 10^{6}\right.$ cells centrifuged at $\left.70 \mathrm{~g}\right)$ onto the cell-coated calcium phosphate carriers (Calcibon ${ }^{\circledR}$, Biomet Merck Biomaterials, Darmstadt, Germany). These biphasic constructs were further cultivated for three weeks (Petersen et al., 2008).

The constructs were implanted in the weight-bearing zone of the medial femoral condyle of the animal from which the cells were originally harvested. Surgery was performed $50 \mathrm{~d}$ after biopsy in all animals. The chondral layer of the implants was trimmed along the edge of the carrier to create a fresh cartilage interface to the native cartilage at the implantation site in the femoral condyle. Constructs were inserted into ø $4.5 \mathrm{~mm} \times 3 \mathrm{~mm}$ drilled holes levelling the articular surface of the cylinder with the adjacent cartilage. All animals were allowed free activity and full weight bearing.

\section{Histology}

Histological sections of cartilage-carriers with the surrounding native cartilage-bone interface of animals sacrificed after 2 weeks $(n=2$; in the following referred to as specimens B and C), 26 weeks ( $n=2$; specimens $\mathrm{D}$ and $\mathrm{E})$ and 52 weeks $(n=2$, specimens F and G) were investigated. Each specimen was dissected out, fixed in freshly prepared $4 \%$ formaldehyde in PBS, pH 7.4, at 4 ${ }^{\circ} \mathrm{C}$, decalcified, embedded in paraffin and cut into $5 \mu \mathrm{m}$ sections in the sagittal plane. One sample of native cartilage (referred to as specimen A) was harvested from animal B close to the implantation site as a reference and treated in the same manner. Since the objective of this study had originally not been part of the animal study, the number of histological sections is limited.

Representative sections of each specimen were stained immuno-histochemically (Petersen et al., 2008). Immunostaining was performed on adjacent tissue sections for collagen type I and type II, respectively. Monoclonal anti-type I collagen (clone I-8H5) and antitype II collagen (clone II-4C11) were purchased from Acris Antibodies $\mathrm{GmbH}$, Herford, Germany. Native cartilage and bone samples were used as positive controls. Negative controls were prepared omitting the primary antibodies. Immunostaining was highly specific, no cross reactions were observed using native cartilage and bone tissue as positive controls.

Epitopes were unmasked for $4 \mathrm{~h}$ at $37{ }^{\circ} \mathrm{C}$ with $1 \mathrm{mg} /$ $\mathrm{mL}$ hyaluronidase in $0.1 \mathrm{M}$ phosphate buffer, $\mathrm{pH} 5.5$; blocking was performed with $10 \%$ serum for $30 \mathrm{~min}$. Primary antibodies $(5 \mu \mathrm{g} / \mathrm{mL})$ were prepared in antibody diluent with background reducing components (Dako Cytomation, Hamburg, Germany). The incubation with primary antibodies was carried out for $16 \mathrm{~h}$ at $4{ }^{\circ} \mathrm{C}$. Subsequently, incubation with biotinylated secondary goat anti-mouse antibody in antibody diluent $(2.5 \mu \mathrm{g} / \mathrm{mL}$, Biozol, Eching, Germany) was carried out for $1 \mathrm{~h}$ at room temperature. Finally, the tissue sections were incubated with Vectastain $\mathrm{ABC}$-alkaline phosphatase reagent for 1 h (Vector Laboratories, Burlingame, CA, USA). Alkaline phosphatase enzyme activity was visualised using the Naphtol-AS-BI-phosphate/New Fuchsin color reaction. Slides were counterstained with Mayer's haemalum 1:1 in distilled water for $30 \mathrm{~s}$ and mounted with Ultra Mount (Dako Cytomation, Hamburg, Germany).

Respective adjacent tissue sections were stained with Sirius Red to enhance the birefringence of the collagen fibres. Prior to staining, the sections were de-waxed in two changes of Rotihistol (Carl Roth GmbH, Karlsruhe, Germany), for 15 min each. Rehydration was performed in a descending ethanol series. After a short immersion in $70 \%$ ethanol, the sections were stained for $24 \mathrm{~h}$ in a 0.5 \% Sirius Red solution (Sirius Red F3B, Dystar GmbH, Leverkusen, Germany). The sections were washed in distilled water for 10 mins and de-hydrated in an ascending ethanol series. Sections were mounted using Roti-Histokitt (Carl Roth $\mathrm{GmbH}$ ) and were covered with a coverslip.

\section{Image acquisition}

Investigation of the collagen fibre network was performed using a polarisation microscope (Nikon Eclipse 80i, Nikon Corp., Tokyo, Japan) in which the specimen is placed between two polarising filters with a $90^{\circ}$ relative orientation to present a dark field in areas lacking birefringence properties. Images were acquired with a digital camera (Nikon DS-5Mc, Nikon Corp.) through lenses of either 40x or 100x magnification, depending on the height of the cartilage layer between the articular surface and the underlying carrier/subchondral bone in the histological section of the specimen. This led to image resolutions of $2.42 \mu \mathrm{m} /$ pixel and $0.97 \mu \mathrm{m} / \mathrm{pixel}$, respectively, allowing clear differentiation of individual chondrocytes. 


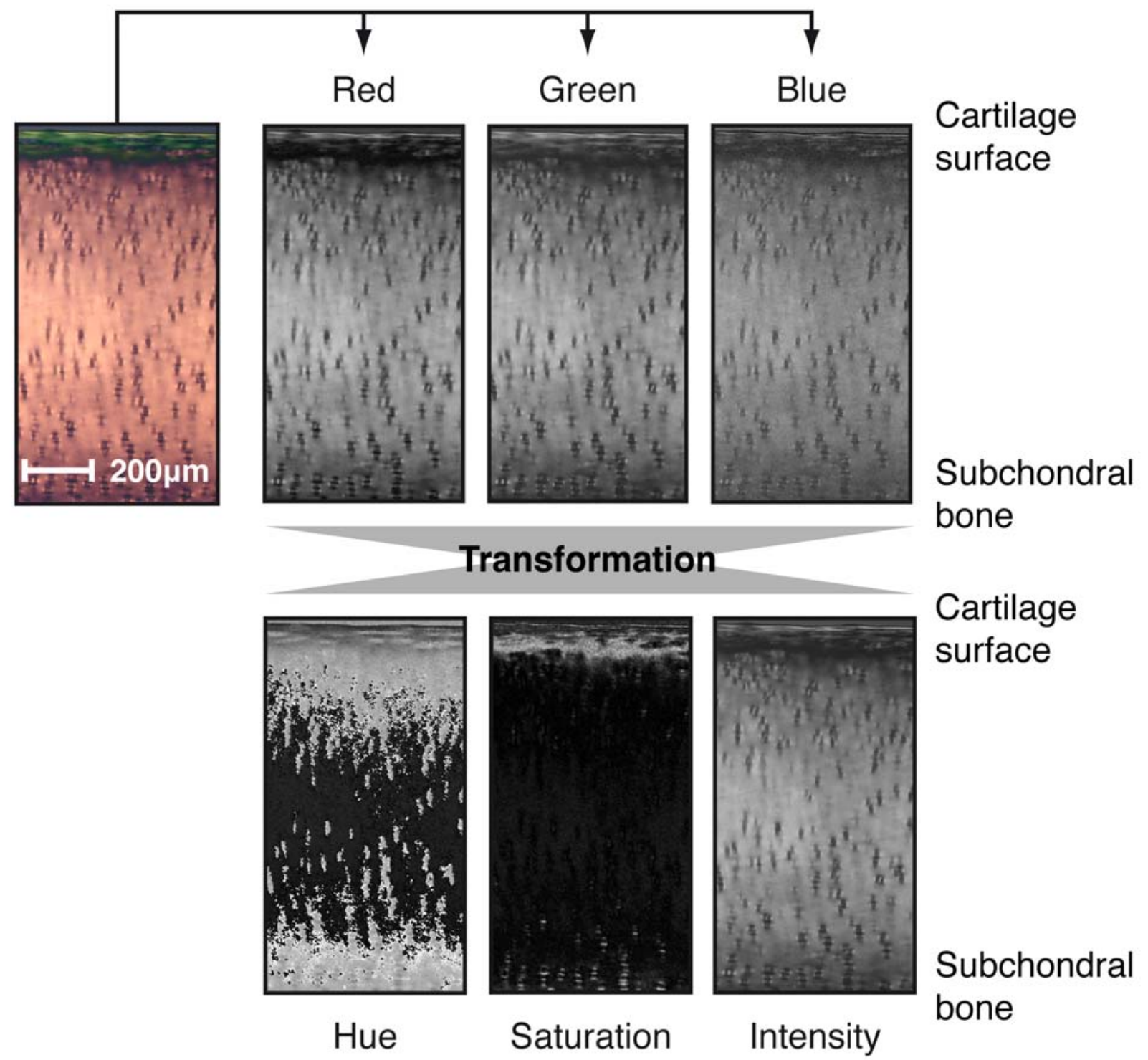

Fig. 1. The digitised colour microscopy image is saved in the RGB colour space. Each pixel consists of a red, green, blue triplet. This information is transformed into the three channels of the HSI colour space being hue, saturation and intensity. These channels are then used for the image analysis on the histological sections.

The sections were centrally positioned on a rotating fixture in the plane of the polarising filters, aligning cartilage surface of the section initially at $45^{\circ}$ with respect to the orientation of the polariser and analyser. Aperture and exposure time were kept constant and additional filters (such as the digital gain) were turned off, to ensure comparability of the images for the digital image analysis procedure. Overexposure of the images was avoided by initially adjusting the exposure time at an angle of the rotation stage for which the maximum birefringence of a specimen occurred.

The images for the analysis were recorded by rotating the histological section in increments of $5^{\circ}$ over a range of $\pm 45^{\circ}$ resulting in 19 images for each specimen. Consequently, every fibre orientation angle from parallel to the joint surface to orthogonal was accounted for. All images were saved using an uncompressed file format (TIFF).

Standard bright field images of the sections stained for collagen type I and type II were taken to assess the collagen distribution with the same microscope in the fluorescence mode. Camera settings were held constant to ensure comparability of the images.

\section{Image processing}

The alignment of the articular surface of the 19 rotated images was performed semi-automatically, using points of interest for a rough alignment followed by fine-tuning with an optimisation algorithm (Avizo, Mercury Systems, MA, USA).

To analyse the collagen fibre orientation, the intensity of each pixel must be determined. Images were therefore transformed from the Red-Green-Blue (RGB) colour space into the Hue-Saturation-Intensity (HSI) space (Gonzalez et al., 2004) (Matlab, The MathWorks, MA, USA). A value of zero for the saturation and intensity channels is equal to a complete infiltration with white light and a value of one represents a complete lack of white light.

For quantitative histological image analysis, the HSI model allows a more direct use of the information 


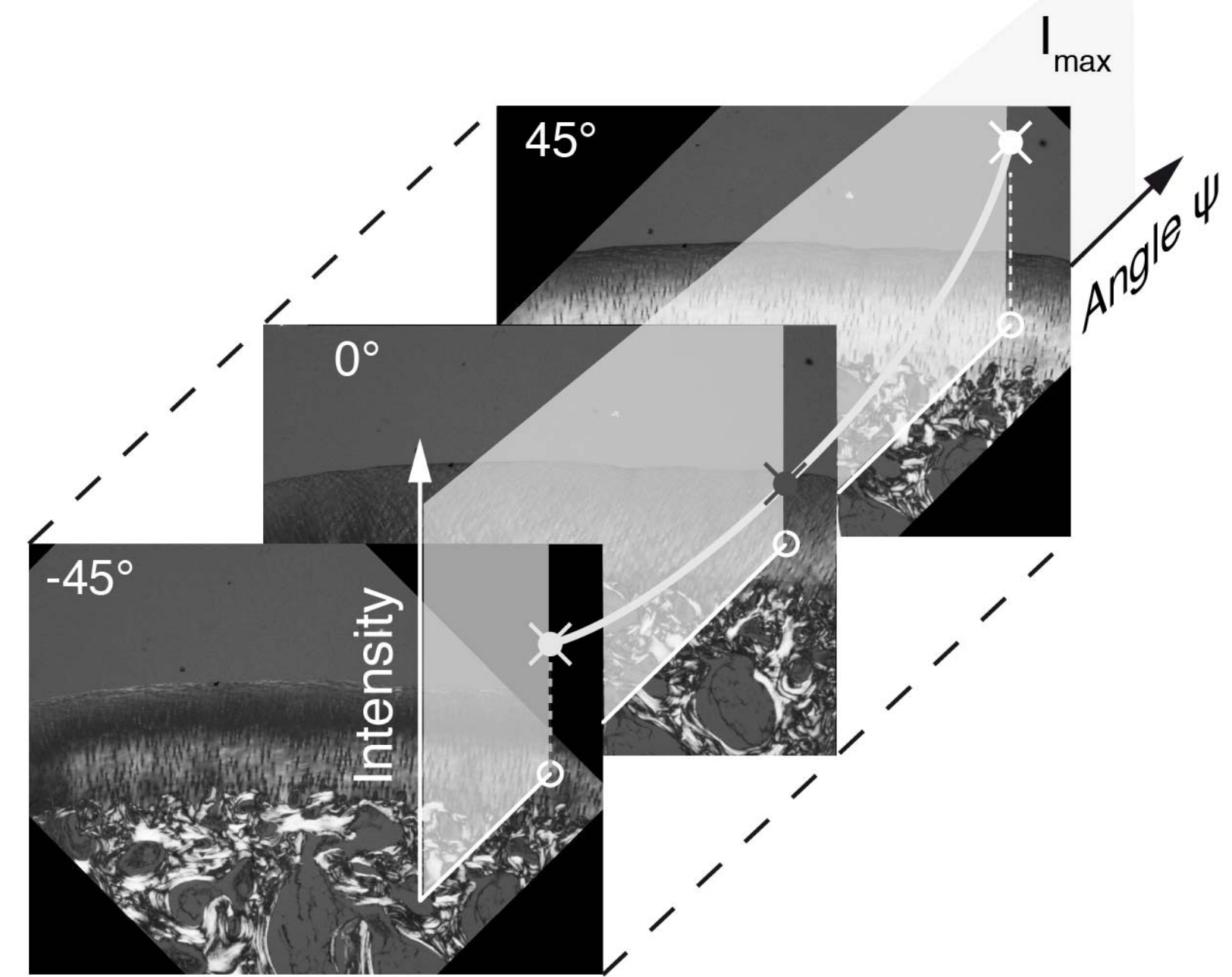

Fig. 2. For each sample, the images taken with different polarisation angles $\psi$ are aligned on top of each other (here, polariser angles $-45^{\circ}, 0^{\circ}$, and $45^{\circ}$ in respect to the cartilage surface of the same histological section are shown) and are saved in a three-dimensional matrix. The first two dimensions define the location of a pixel with respect to the histological section; the third dimension defines its intensity in respect to the polarisation angle $\psi$. For the determination of the dominant collagen fibre orientation at a location/pixel, the maximum intensity Imax along the third dimension is determined. Repeating this for all pixels allows derivation of the orientation of the collagen fibre network of a specimen's histological section.

contained within the pixels of the image than the RGB model, since only one value has to be considered to perform the analysis. For example, for a histological stain that is investigated by bright field microscopy, it is the Saturation channel that provides the local concentration of the stain relative to the other portions of the specimen. When using fluorescence microscopy, the Intensity channel will provide this information. In addition, filtering out information on the basis of a certain wavelength, i.e. colour, can easily be done by using the information contained in the Hue channel. In the following, the Intensity channel is used for the analysis of the collagen fibre orientation (Fig. 1) while the Saturation channel facilitates the analysis of the collagen distribution within the histological sections.

The pixels, stacked virtually on top of each other in an array data structure, have intensity values between zero and one (Fig. 2). The maximum intensity of a pixel correlates with the birefringence of the specimen. Therefore, knowing the maximum intensity of a pixel, the dominant orientation of the collagen fibres can be derived from the angle of orientation of the histological section relative to the polariser and analyser. Due to background intensity noise, birefringence was only considered if rotating the stage caused a change of at least a 0.05 in intensity. The accuracy of determining the angle describing the dominant fibre orientation is dependent on the incremental rotation of the stage $\left( \pm 2.5^{\circ}\right.$ in this study). The dominant collagen fibre orientation for each pixel due to the combined analysis of the images can subsequently be displayed as a pseudo colour image.

In a further analysis, the same information was used to derive the dominant collagen fibre orientation as a function of the relative depth of the cartilage tissue. Because the surface of the cartilage is not even, the shape of the surface 


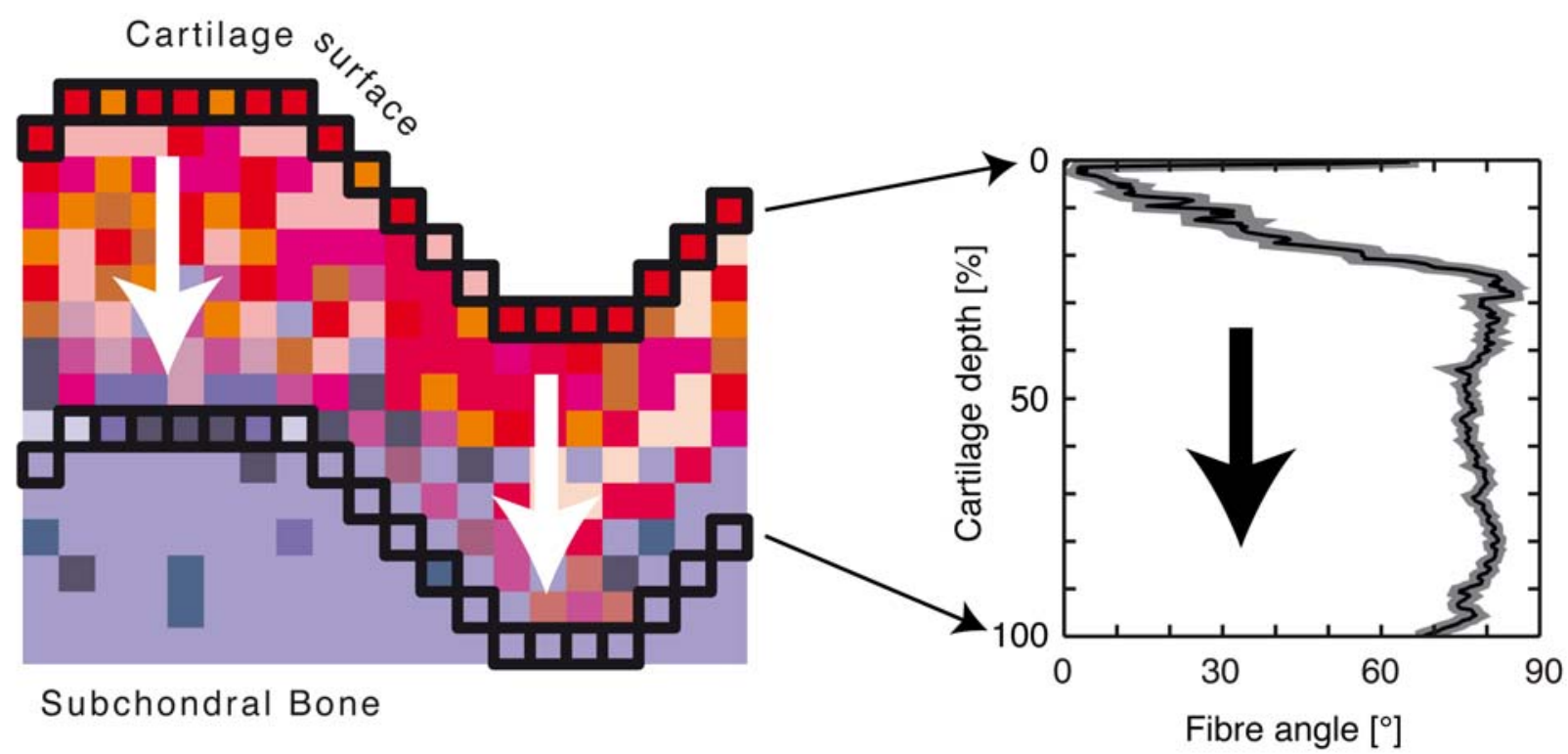

Fig. 3. Calculation of the fibre orientation as a function of the cartilage depth along the surface profile. The surface profile was moved downwards pixel-by-pixel from the articular surface to the border of the subchondral bone. During each step, the mean value for the fibre angle along the profile as well as its standard error is calculated and visualised in a graph showing the fibre angle versus cartilage depth.

was taken to be the base line from which the mean and the standard error of the fibre orientation were acquired (Fig. 3). Since birefringence is not present in the region above the cartilage surface, where the histological section ends, the surface profile can easily be identified due to the constant intensity values of the pixels over the 19 images (Matlab). The profile obtained is then moved pixel-by-pixel in a depth-wise direction from the cartilage surface down to the subchondral bone. For each step from the cartilage surface down to the border of the subchondral bone, the mean angle and standard error were calculated along the profile. Isotropic pixels were excluded from the latter calculation steps.

The immuno-histochemical staining procedure does not allow for the comparison of the collagen content among different histological sections but can be used for the visualisation of the relative collagen type I and type II distributions within the histological sections. The amount of stain correlates with the Saturation channel (Fig. 4) allowing visualisation of the collagen distribution as a pseudo colour image.

\section{Results}

\section{Collagen fibre orientation of native cartilage}

The collagen network of native articular cartilage showed anisotropic properties (Figs. 5 and 6; specimen A). Within the superficial zone, the collagen fibres are oriented predominantly parallel to the surface up to a depth of approximately $30 \mu \mathrm{m}$. In the adjacent transitional zone, the fibre orientation becomes isotropic presenting as a approximately $30 \mu \mathrm{m}$ wide band oriented parallel to the joint surface. In the deep zone down to the subchondral bone, the collagen network becomes anisotropic again, aligned perpendicularly to the joint surface (Fig. 5; specimen A). This change can also be seen in the graph, indicating the fibre orientation at the respective cartilage tissue depth (Fig. 6; specimen A). The cartilage depth is presented normalised to the total cartilage height of each specimen, where $0 \%$ corresponds to the articular surface and $100 \%$ to the transitional zone of the subchondral bone. In addition, the alignment of the fibres in the deep zone of the native cartilage is very consistent as can be seen by the low standard error (grey area beneath the line).

\section{Collagen fibre orientation of implanted tissue engineered cartilage}

The histological sections of the tissue engineered cartilage retrieved two weeks after implantation showed first signs of an aligning collagen fibre network (Figs. 5 and 6; specimens B and C). The collagen fibres in the superficial zone are orientated parallel to the surface down to a relative depth of $10 \%$ to $20 \%$, similar to the native tissue (12\%) (Fig. 6). However, the absolute depth value for the fibres oriented parallel to the cartilage surface (about $100 \mu \mathrm{m}$ ) differs from the value found in native tissue. The transitional and deep zones have a predominantly isotropic fibre network. In the deep zone of specimen B, there are already some small regions of vertically oriented fibres apparent. The majority of the intercellular space within the deep zone displays an isotropic fibre orientation. In contrast, the fibres surrounding the chondrocytes already show anisotropic characteristics (Figs. 5, 6 and 7; specimens $\mathrm{B}$ and $\mathrm{C}$ ), running tangentially around the periphery of the cell lacunae.

In the deep zone directly above the calcium phosphate carrier of the implants retrieved after two weeks, the fibres of the tissue-engineered cartilage are orientated parallel with respect to the cartilage surface, similar to those in 


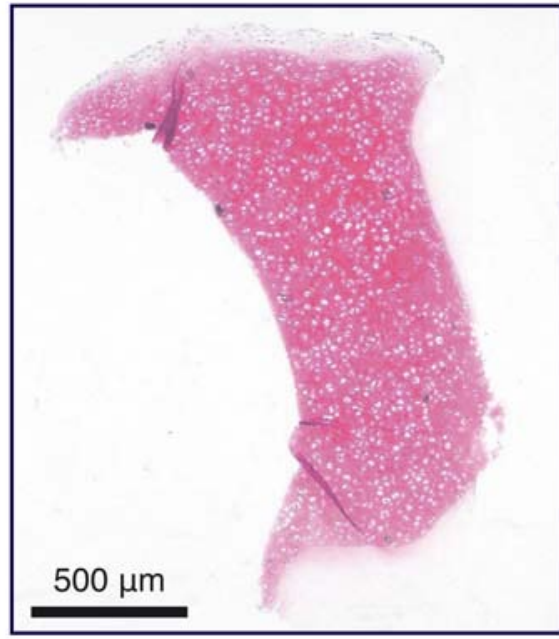

RGB color space

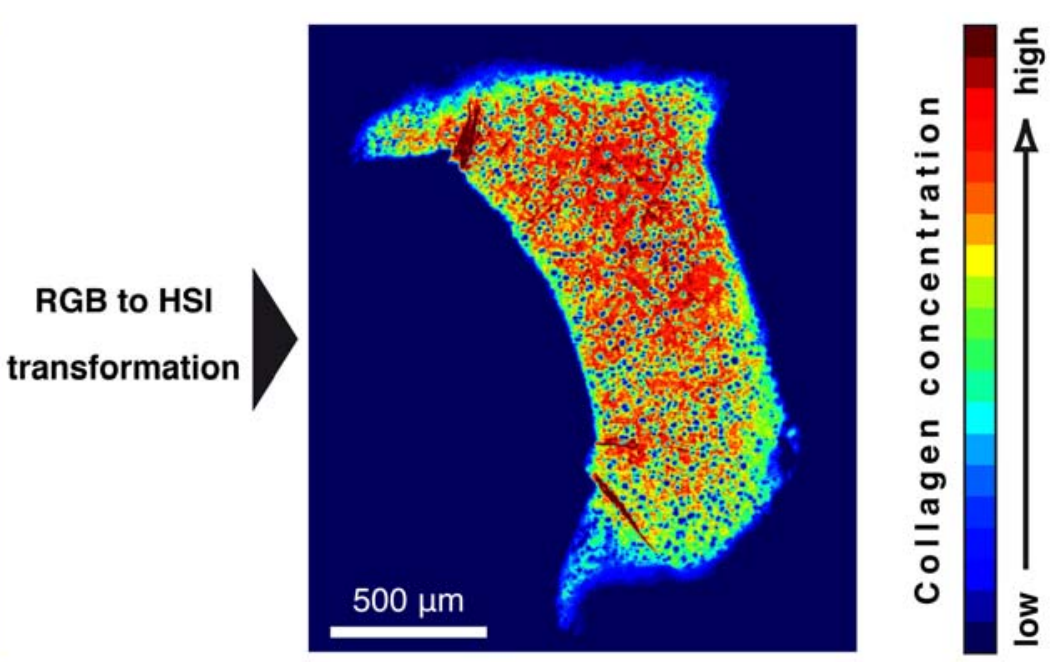

HSI color space: saturation

Fig. 4. A brightfield microscopy image of a histological section is transformed from the RGB colour space to the HSI colour space. The saturation channel being dependent on the relative amount of collagen in the image of a histological section is displayed as a pseudo colour image to visualise the relative collagen distribution throughout the tissue sample.

the superficial zone (Figs. 5 and 6; specimens B and C). The analysis of the fibre angle as a function of cartilage depth demonstrates an irregular orientation in the 2 week tissue-engineered implants $\mathrm{B}$ and $\mathrm{C}$ compared to the homogeneous change in fibre orientation of the native tissue $\mathrm{A}$. The high standard error for $\mathrm{B}$ and $\mathrm{C}$ in comparison to specimen $\mathrm{A}$, emphasises the irregular fibre orientation in these specimens.

After 26 weeks, the collagen fibres of the histological sections constitute a highly structured network (Figs. 5, and 6; specimens D and E). At the joint surface, the collagen fibres are orientated parallel with respect to the cartilage surface up to a depth of 25-100 $\mu \mathrm{m}$. In the transitional and deep zones, the orientation changes to become predominantly diagonal (Figs. 5 and 6; specimen E) or vertical (Figs. 5 and 6; specimen D). In specimen D, the cartilage was still in contact with the calcium phosphate carrier and the collagen fibres developed a fibre orientation parallel to the surface that extended up to $400 \mu \mathrm{m}$ into the deep zone of the tissue. In specimen E, a layer of bone is present between the tissue-engineered cartilage and the carrier. Here, the collagen fibres retained their diagonalto-vertical orientation, even at the cartilage-bone interface. The analysis of the fibre orientation profile after 26 weeks shows a more uniformly oriented network than for the specimens at 2 weeks. However, the tissue-engineered cartilage samples still differ markedly from the native cartilage. Especially in the deep zone, the tissue-engineered cartilage fibres tend to be oriented more parallel with respect to the cartilage surface.

After 52 weeks (Figs. 5 and 6; specimens F and G) the collagen fibre network is similar to native cartilage, although not quite as homogeneous (Figs. 5 and 6; specimen A). The superficial zone of both implants exhibits collagen fibres oriented parallel to the joint surface. However, in implant $\mathrm{G}$ this zone is only about $10 \mu \mathrm{m}$ thick and does not completely cover the joint surface. In the transitional zone the fibre orientation is either isotropic (Figs. 5 and 6; specimen F) or vertical (Figs. 5 and 6; specimen G).
In both specimens, a layer of subchondral bone had been developed between the carrier and the cartilage tissue. The similarity in fibre orientation between the implants (Figs. 5 and 6; specimens $\mathrm{F}$ and $\mathrm{G}$ ) and the native cartilage (Figs. 5 and 6; specimen A) can easily be seen from the similar trend for the collagen fibre orientation depth profiles (Fig. 6). However, in the tissue-engineered cartilage, the change in fibre orientation from the superficial to the deep zone occurs with a gradient that is less steep than that for native cartilage.

\section{Collagen distribution in native cartilage}

No collagen type I was found in the histological section of native cartilage tissue but it was present in the subchondral bone (Fig. 8; specimen A). The distribution of collagen type II shows a consistent pattern throughout the entire depth of the cartilaginous tissue.

\section{Collagen distribution in implanted tissue}

In contrast to the native tissue, both collagen types were found throughout the tissue-engineered cartilage for the 2 weeks specimens (Fig. 8; specimens B and C). For the 26 week tissue (Fig. 8; specimens D and E), implant D has developed a triangular region containing collagen type I directly above the calcium phosphate carrier. There is little collagen type I visible between the tip of the triangle and the joint surface. Directly beneath the cartilage surface, a strip of tissue containing collagen type I is present, reaching $50 \mu \mathrm{m}$ into the tissue. In particular, a narrow strip staining positively for collagen type II is observed parallel to the right side of the triangle containing mostly collagen type I. In contrast, implant $\mathrm{E}$ shows a collagen distribution that is more similar to the two week old specimens B and C.

At 52 weeks, there is very little collagen type I is found within the tissue-engineered cartilage (Fig. 8; specimens F and G), but collagen type II is present throughout the tissue. There is a maximum staining intensity close to the articular surface, similarly to the native cartilage (Fig. 8; specimen A). 
Native cartilage
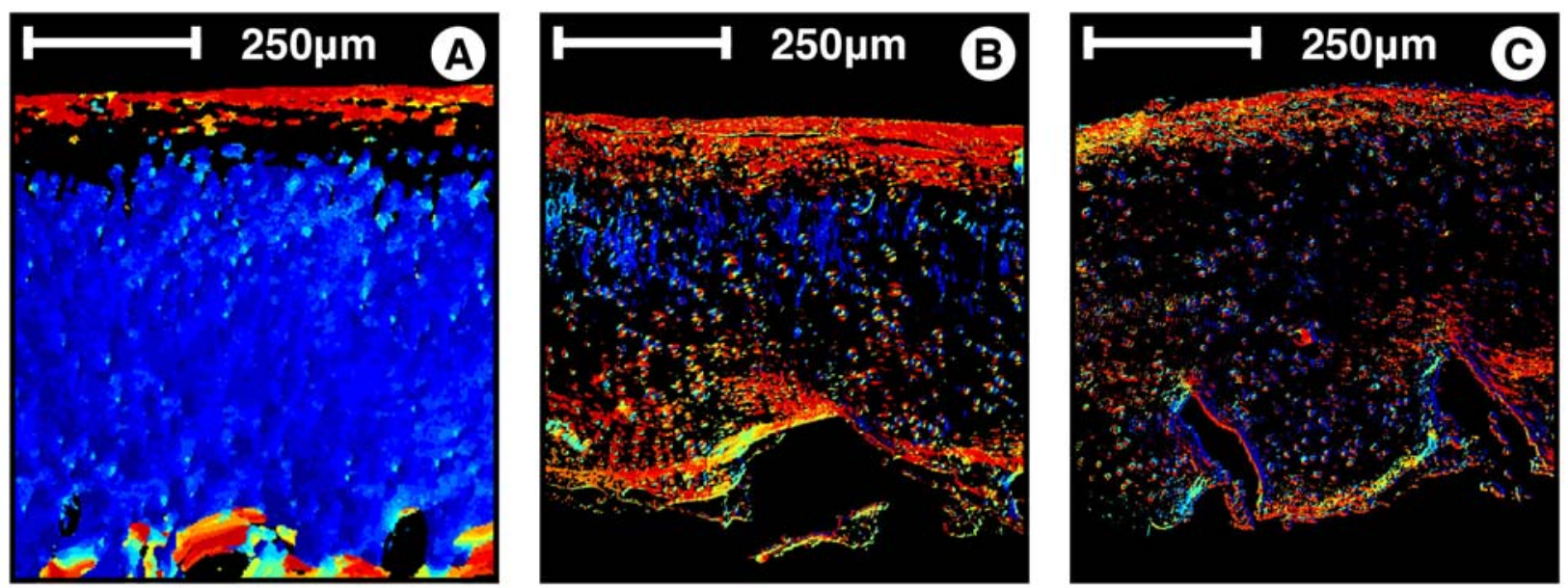

Tissue engineered cartilage after 26 weeks

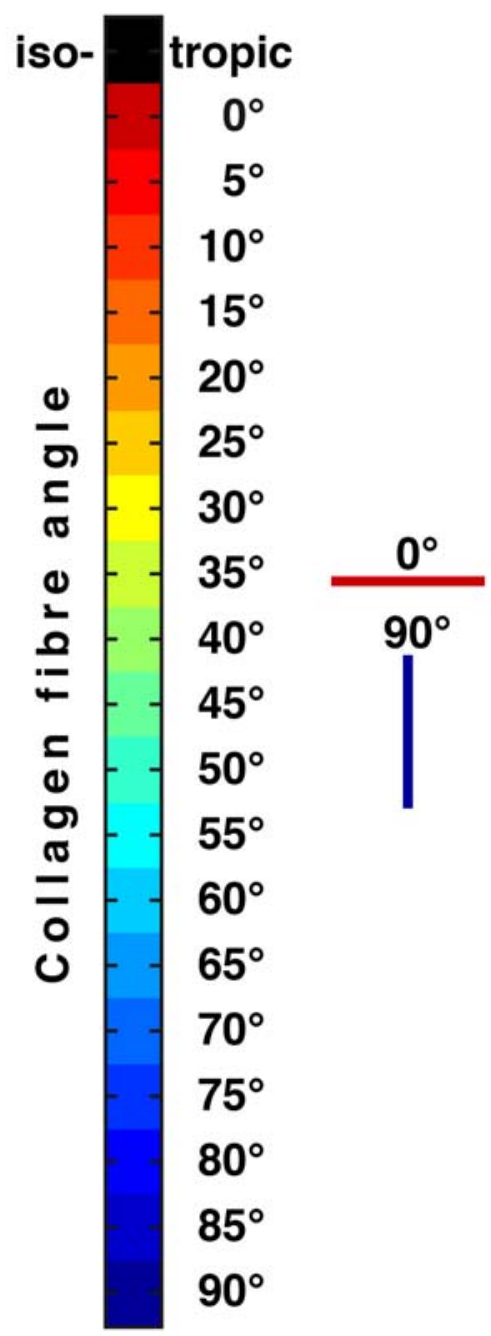

Tissue engineered cartilage after 2 weeks
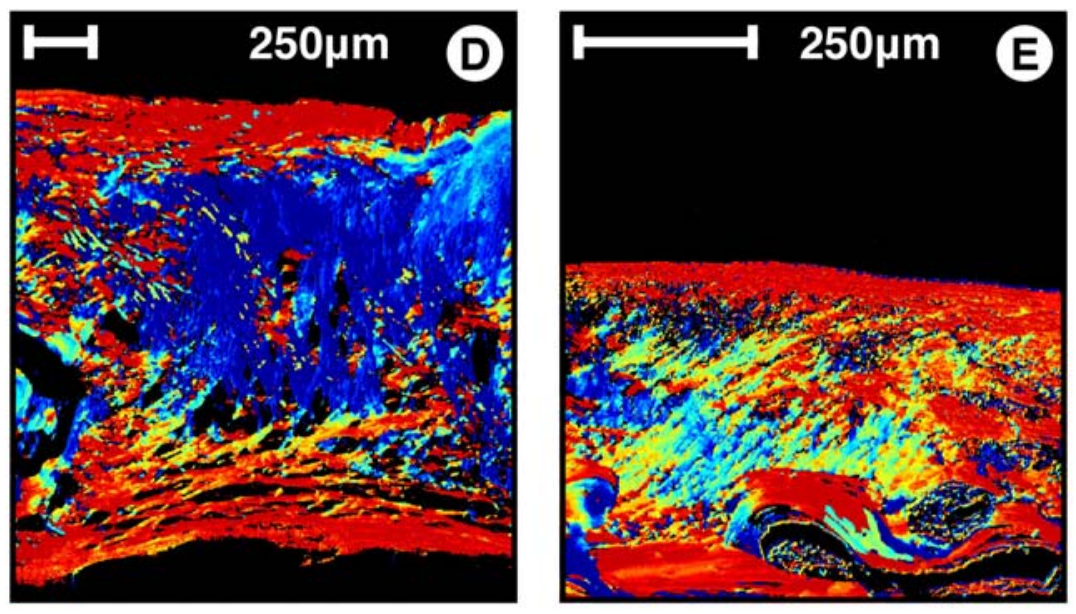

Tissue engineered cartilage after 52 weeks
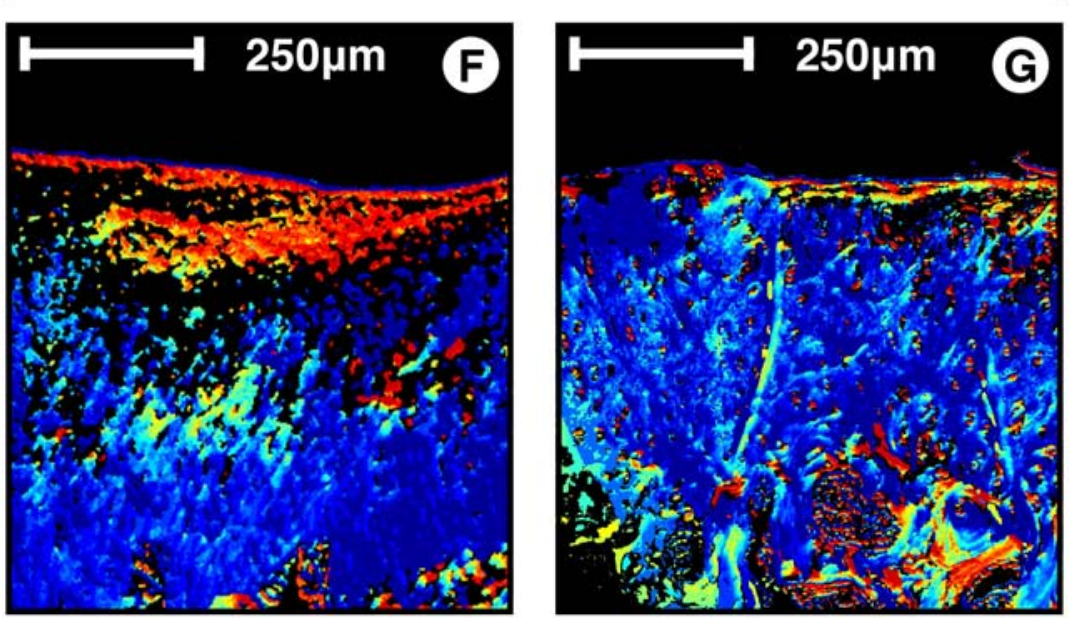

Fig. 5. Collagen fibre network development within the tissue-engineered cartilage specimens (B, C: implanted for 2 weeks; D, E: implanted for 26 weeks; F, G: implanted for 52 weeks) compared to native cartilage (A). An angle of $0^{\circ}$, visualised in red, represents collagen fibres oriented parallel to the cartilage surface, whereas an angle of $90^{\circ}$, visualised in blue, denotes collagen fibres that are oriented perpendicular to the cartilage surface. 

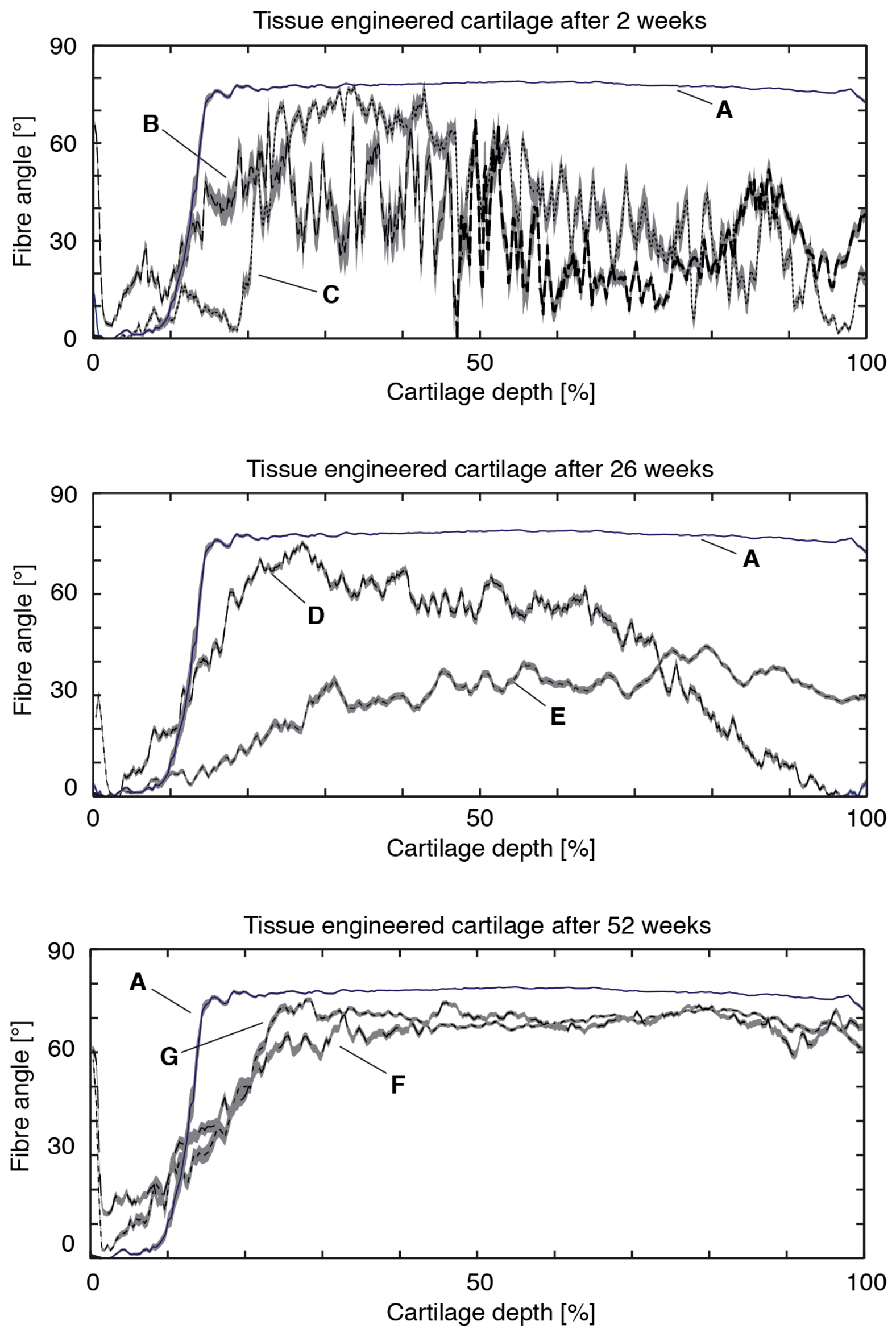

Fig. 6. The depth profiles (mean shown as a line \pm standard error shown as a grey band) display the fibre orientation along the relative depth of the cartilage for the tissue-engineered specimens (B, C: implanted for 2 weeks; D, E: implanted for 26 weeks; F, G: implanted for 52 weeks) compared to the native cartilage specimen (A). 

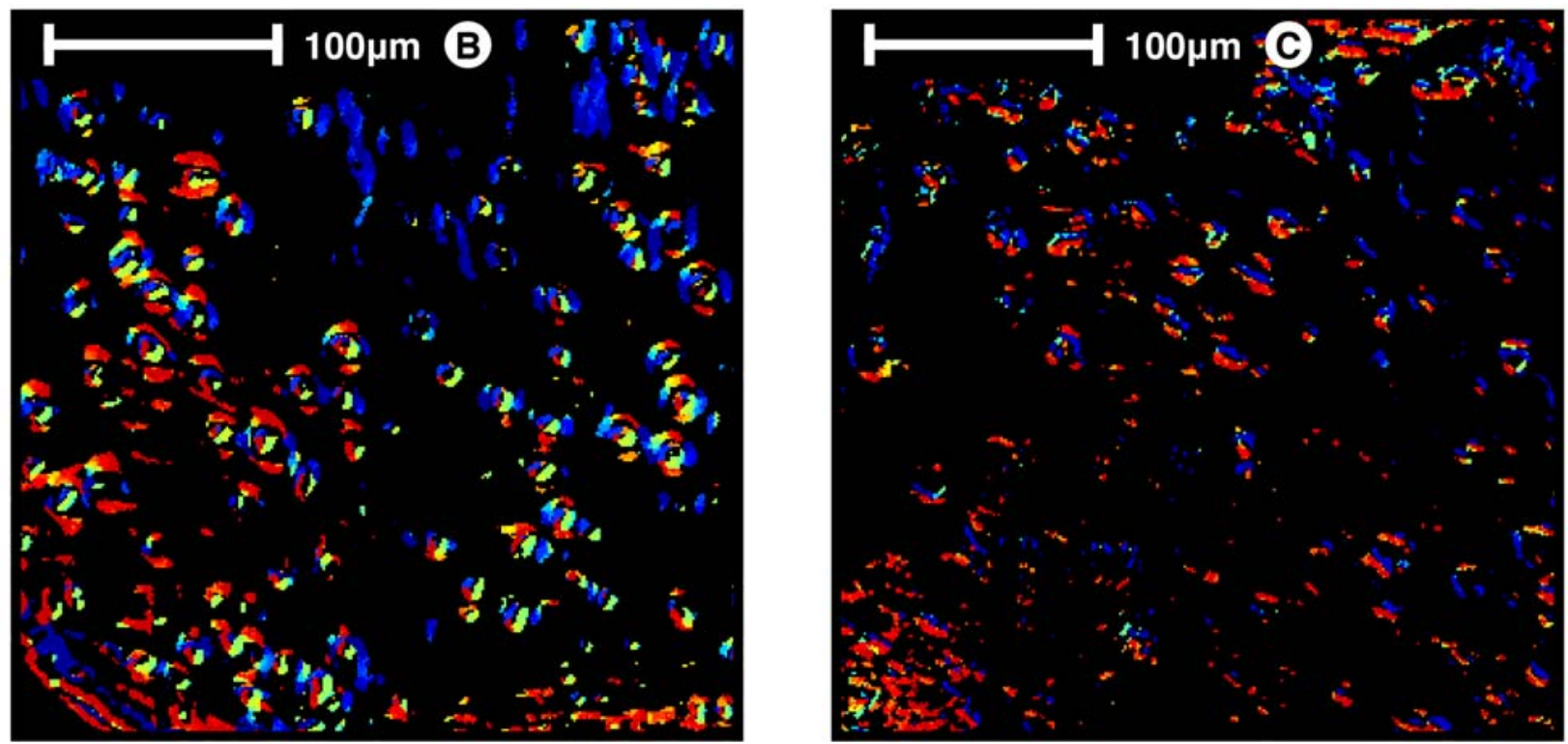

Fig. 7. Detailed view of the collagen fibre network in the deep zone of the cartilage specimens B and C implanted for 2 weeks. Although the tissue generally shows isotropic properties (also see Fig. 6), collagen fibres appear to be oriented around single cells of the tissue.

\section{Discussion}

Applying polarisation microscopy and the digital image analysis method developed for this study, it was possible to determine the fibre orientation at each location within each single histological section. To our knowledge, the development of the collagen fibre orientation over several time points post implantation of implanted tissue-engineered cartilage has not been shown before. Furthermore, the determination of the simultaneous collagen type distribution allows determination of the relationship between the fibre orientation and collagen type present.

The orientation of the collagen fibre network within the native tissue corresponds to the literature (Benninghoff, 1925; Hughes et al., 2005; Rieppo et al., 2009). This can be viewed as an indication that the image analysis method developed in this study successfully allows determination of the spatial fibre orientation in cartilage. The results for the tissue-engineered constructs demonstrated that the method can also be used to detect a less developed orientation of collagen fibres which cause less birefringence.

\section{Collagen fibre orientation}

The first region to develop an anisotropic structure within the tissue-engineered cartilage was the superficial zone. This agrees with the observation of Hughes et al. (2005), who investigated the development of the fibre network of mice in an age range from one day to eight months. They recorded fibres oriented parallel to the surface starting from five days after birth. Before that no anisotropy was observed. They proposed that the development of an anisotropic fibre network may be initiated by joint loading. Based on the result of the current study, the anisotropic properties of the superficial zone already after two weeks of implantation suggest that the cartilage implants were indeed responding to joint loading. This is supported by the observation that the animals showed normal activity post operatively (data not shown).

Although one of the two-week implants showed the first signs of a vertical orientation of the bulk collagen fibre network, the transitional and deep zones mostly displayed an isotropic orientation of the collagen fibres. However, in these zones a fibre network orientation was already detectable in the immediate vicinity of the chondrocytes with fibres running circumferentially around the periphery of the cells. This local orientation pattern, of continuously varying fibre angles, leads to an exaggerated standard error over the tissue section as a whole. Consequently, the angular profile over the cartilage depth shows a rather irregular behaviour with a comparably high standard error for these two specimens.

The oriented fibre matrix surrounding the cells is no longer found at 26 weeks. This might indicate that the birefringence effect of the interterritorial matrix is dominant, covering up the birefringence effect caused by the local fibre network around the cells. In view of studies showing that the territorial collagen fibre network is formed circumferentially around the chondrocytes of adult animals (Hunziker et al., 1997; Poole et al., 1987; Poole et al., 1984), this seems to be more likely than the disappearance of the cell-enclosing network.

The fibre network was still anisotropic throughout the depth of the tissue-engineered cartilage after 26 weeks in vivo, with the depth profile of the predominant collagen fibre orientation markedly different from the profile of the native cartilage. In respect to sample E, there is subchondral bone present underneath the implant's tissueengineered cartilage, with the bone-cartilage boundary of the implant zone being relatively close to the articular surface. Consequently, there is a reduced height of the overlying tissue compared to the other samples. In other 


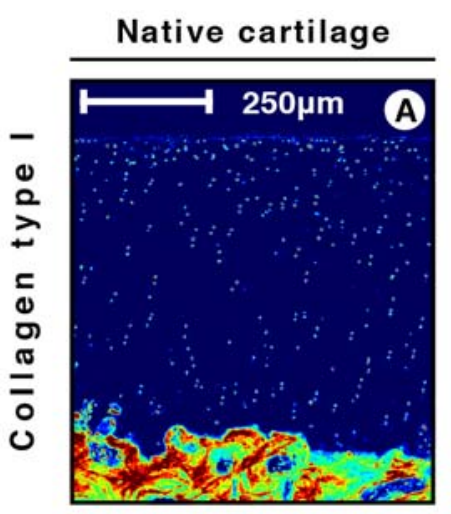

Tissue engineered cartilage after 2 weeks
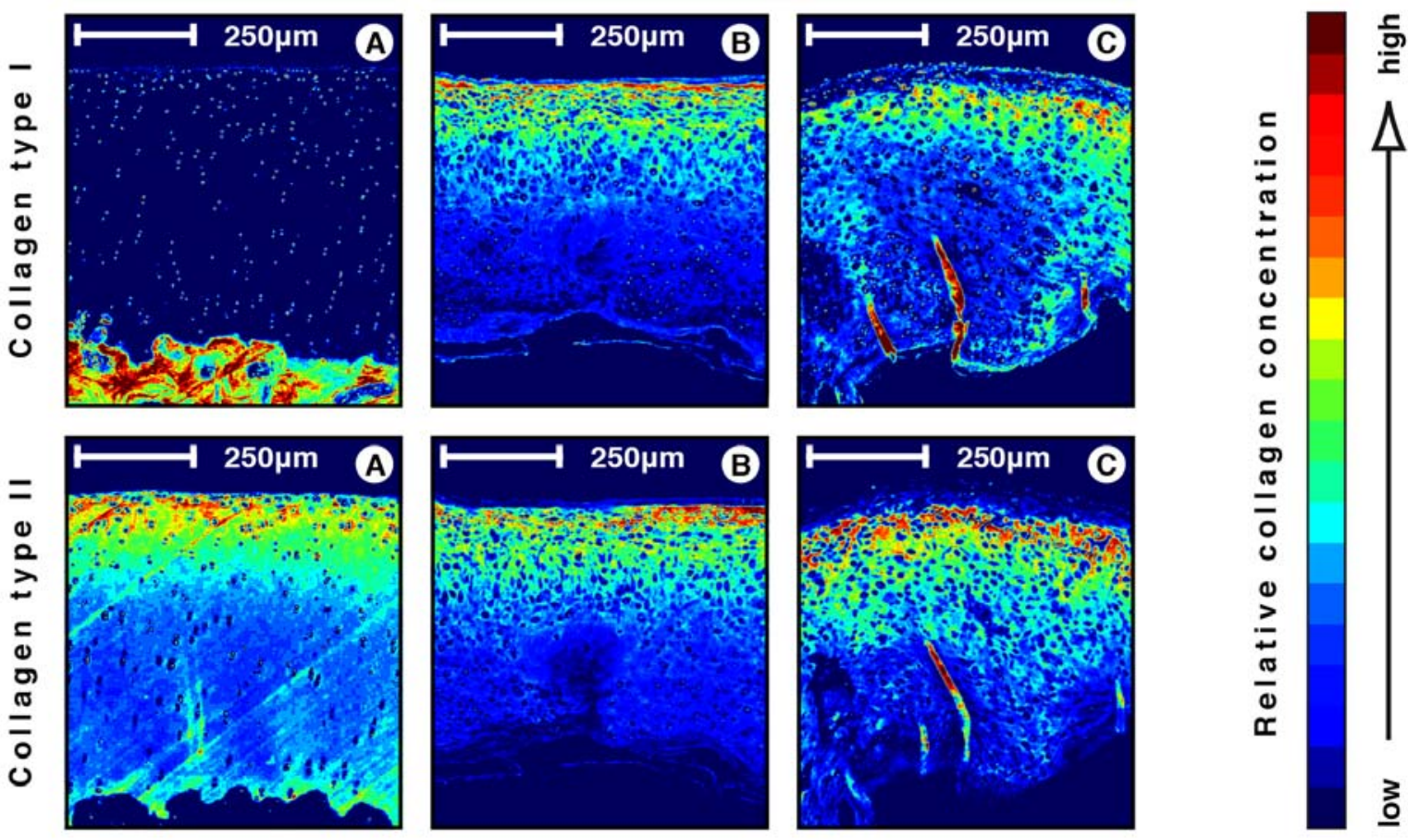

Tissue engineered cartilage after 26 weeks
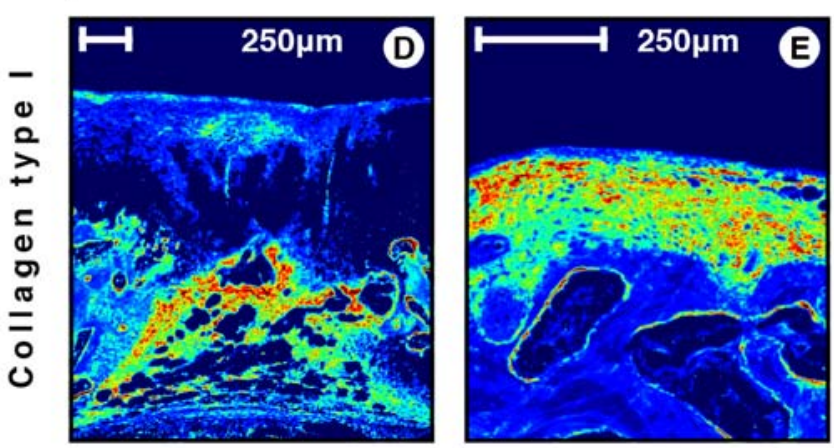

Tissue engineered cartilage after 52 weeks
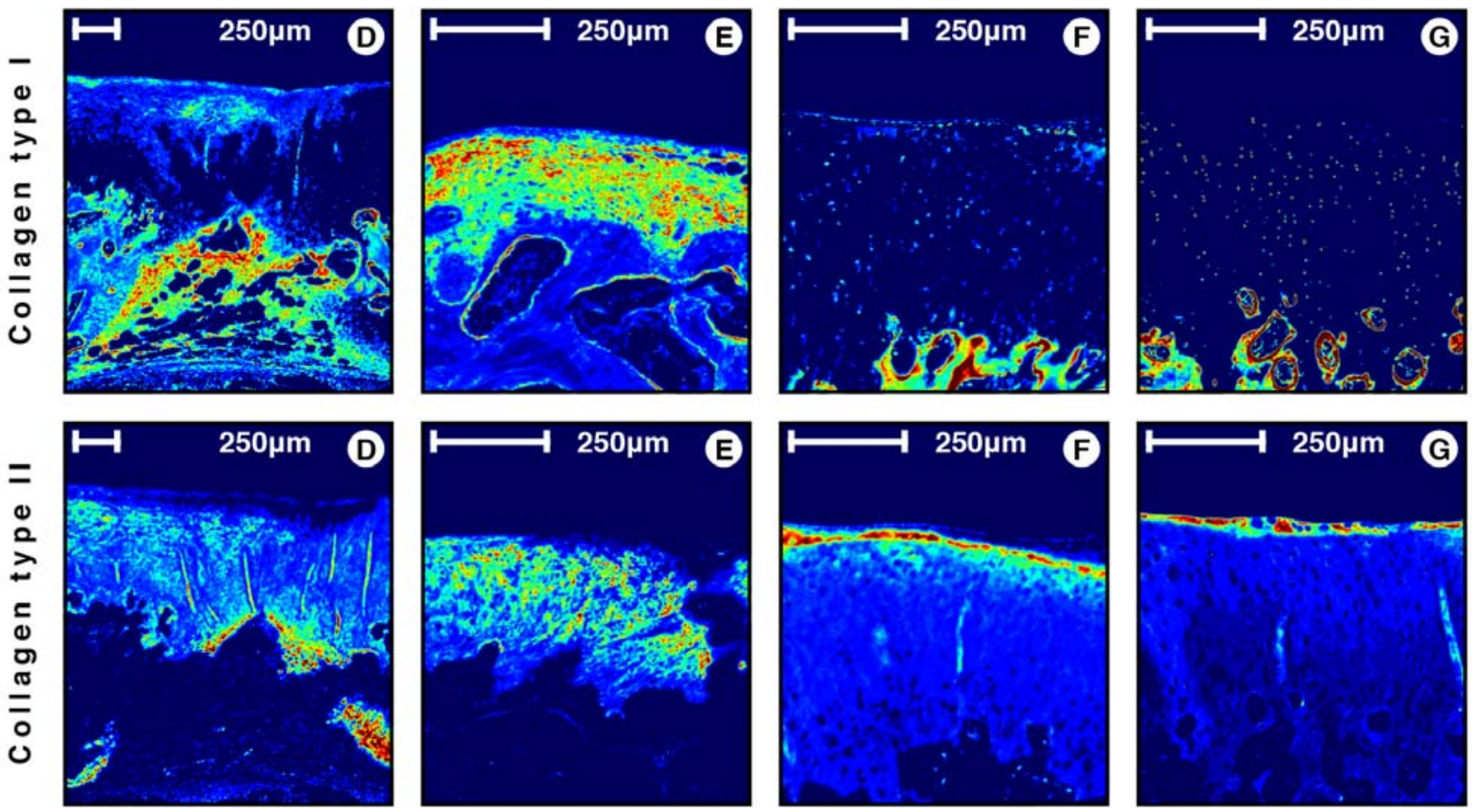

Fig. 8. Relative collagen distribution of collagen type I and type II in native and tissue-engineered cartilage samples after 2, 26 and 52 weeks. With increasing duration of implantation, the relative collagen distribution in the tissueengineered cartilage increasingly resembles the relative collagen distribution in native cartilage.

words, the regenerating bone tissue in sample $\mathrm{D}$ as well as in the 52 week samples $\mathrm{F}$ and $\mathrm{G}$, even though not fully closed, is formed further away from the articular surface. It seems that the comparatively thin layer of tissue above the newly formed subchondral bone in sample E leads to a load distribution or load transfer throughout the tissue into the subchondral bone that affected the remodelling process of the collagen fibre network, resulting in a predominantly diagonal orientation of the collagen fibres with respect to the articular surface.

After 52 weeks, the implanted samples nearly resemble native cartilage. Although lacking the homogeneous appearance of native cartilage, especially near the articular surface, the pseudo colour images, as well as the depth profiles of the collagen fibre orientation, are quite similar for implanted and native cartilage. 
On the basis of split line patterns in native cartilage, Pauwels et al. (1980) proposed that the orientation of the collagen fibres at the articular surface may result from local tensile strains occurring during joint loading. The observation that the superficial zone of the implanted tissue-engineered cartilage is the first zone to develop a fibre orientation might reflect a greater strain magnitude at the surface than in the transitional and deep zones. This could be clarified by performing a similar study with samples implanted in non-load-bearing regions.

The orientation of collagen type I fibres parallel to the carrier surface for younger samples B, C and D contrasts with a perpendicular alignment in older samples $\mathrm{E}, \mathrm{F}$ and $\mathrm{G}$, in which bone has grown between the carrier and cartilage, and this might also have a mechanically basis. In the younger samples, the cartilage is not well bonded to the carrier, which may allow tensile strains to be generated parallel to the carrier surface. In the older samples, the new bone at the interface may limit strains in the tangential direction, with the perpendicular fibre orientation rather due to bone modelling processes.

\section{Collagen type distribution}

In most samples both collagen types I and II were present during the development of an anisotropic collagen fibre network in the tissue-engineered cartilage. The exceptions were the two samples with the longest implantation time of 52 weeks with nearly only collagen type II, similar to native cartilage. Although the tissue-engineered cartilage contained collagen type I and type II prior to implantation and at 2 weeks after implantation, a shift towards predominant collagen type II expression occurred after 26 weeks (sample D) and 52 weeks (samples F and G). This is consistent with the findings of Roberts et al. (2009), who assessed the distribution of procollagen type IIA, collagen type II and collagen type I following autologous chondrocyte implantation in the human knee joint. They found that the percentage of area staining for type I collagen was the least three or more years after treatment. In contrast, the area staining positively for type II collagen was increased for up to three years or more post-treatment. Simultaneously with the development of the anisotropic fibre orientation, collagen type I disappears. However, similar to the difference in development of the collagen fibre network, in sample E a shift towards collagen type II fails to appear, probably also caused or influenced by the loading of the tissue. In addition, collagen type I fibres oriented parallel to the carrier were observed in samples $\mathrm{B}, \mathrm{C}$ and $\mathrm{D}$ in the region directly above the carrier. The calcium phosphate carrier probably causes an increased collagen type I content, due to its similarity with bone, in which this collagen type predominates.

It has to be noted that although the general collagen distribution in the native cartilage, and in most of the tissue-engineered samples, match observations made in the literature (Muir et al., 1970), the staining methods applied cannot be used to quantify collagen content, since epitopes are affected by factors such as tissue density or masking by GAG molecules. Furthermore, at the border of the histological sections, parts of the collagen fibres might have become detached during preparation of the histological sections, leading to low collagen content observed at the articular surfaces. This may indicate an impaired integrity of the tissue-engineered cartilage in comparison to the native tissue, since such an effect was not observed in the native tissue.

\section{Conclusions}

A new, easy-to-use and standardised method to process digitised microscopy images of histological sections was developed to determine the fibre orientation of native porcine cartilage, as well as the collagen fibre network of tissue-engineered cartilage that had been implanted over periods of 2, 26 and 52 weeks. It was demonstrated that tissue-engineered cartilage has the potential to develop an orientation of the collagen fibre network similar to native cartilage.

\section{Acknowledgments}

The authors gratefully acknowledge G. Mielke for staining the histological sections, N. Bishop for proof-reading, and J. P. Petersen, P. Ueblacker, C. Goepfert, P. Adamietz, K. Baumbach, A. Stork, J. M. Rueger, R. Poertner, M. Amling and N. M. Meenen for providing the histological sections from the animal study.

\section{References}

Agnesi F, Amrami KK, Frigo CA, Kaufman KR (2008) Comparison of cartilage thickness with radiologic grade of knee osteoarthritis. Skeletal Radiol 37: 639-643.

Alhadlaq HA, Xia Y, Moody JB, Matyas JR (2004) Detecting structural changes in early experimental osteoarthritis of tibial cartilage by microscopic magnetic resonance imaging and polarised light microscopy. Ann Rheum Dis 63: 709-717.

Bae WC, Temple MM, Amiel D, Coutts RD, Niederauer GG, Sah RL (2003) Indentation testing of human cartilage: sensitivity to articular surface degeneration. Arthritis Rheum 48: 3382-3394.

Baumbach K, Petersen JP, Ueblacker P, Schröder J, Goepfert C, Stork A, Rueger JM, Amling M, Meenen NM (2008) The fate of osteochondral grafts after autologous osteochondral transplantation: a one-year follow-up study in a minipig model. Arch Orthop Trauma Surg 128: 12551263.

BenninghoffA (1925) Form und Bau der Gelenkknorpel in ihren Beziehungen zur Funktion. Zweiter Teil: Der Aufbau des Gelenkknorpels in seinen Beziehungen zur Funktion. (Engl.: Shape and structure of articular cartilage in respect to function. Part 2: The structure of articular cartilage in respect to its function.) Z Zellforsch Mikrosk Anat 2: 783-862.

Bhosale AM, Richardson JB (2008) Articular cartilage: structure, injuries and review of management. Br Med Bull 87: 77-95.

Buckwalter JA, Mankin HJ, Mow VC (2000) Articular cartilage repair and osteoarthritis. In: Orthopaedic Basic Science: Biology and Biomechanics of the Musculoskeletal System (T.A. Einhorn, S.R. Simon, J.A. Buckwalter, eds), 
American Academy of Orthopaedic Surgeons, Rosemont, Ill., pp 472-488.

Gonzalez RC, Woods RE, Eddins SL (2004) Color image processing. In: Digital image processing using MATLAB (R.C. Gonzalez, R.E. Woods, S.L. Eddins, eds), Pearson/Prentice Hall, Upper Saddle River, NJ, pp194-241.

Gründer W (2006) MRI assessment of cartilage ultrastructure. NMR Biomed 19: 855-876.

Hughes LC, Archer CW, ap Gwynn I (2005) The ultrastructure of mouse articular cartilage: collagen orientation and implications for tissue functionality. A polarised light and scanning electron microscope study and review. Eur Cell Mater 9: 68-84.

Hunziker EB, Michel M, Studer D (1997) Ultrastructure of adult human articular cartilage matrix after cryotechnical processing. Microsc Res Tech 37: 271-284.

Hyttinen MM, Holopainen J, van Weeren PR, Firth EC, Helminen HJ, Brama PA (2009) Changes in collagen fibril network organization and proteoglycan distribution in equine articular cartilage during maturation and growth. J Anat 215: 584-591.

Julkunen P, Harjula T, Iivarinen J, Marjanen J, Seppanen K, Narhi T, Arokoski J, Lammi MJ, Brama PA, Jurvelin JS, Helminen HJ (2009) Biomechanical, biochemical and structural correlations in immature and mature rabbit articular cartilage. Osteoarthritis Cartilage 17: 1628-1638.

Magnussen RA, Dunn WR, Carey JL, Spindler KP (2008) Treatment of focal articular cartilage defects in the knee: a systematic review. Clin Orthop Relat Res 466: 952-962.

Muir H, Bullough P, Maroudas A (1970) The distribution of collagen in human articular cartilage with some of its physiological implications. J Bone Joint Surg Br 52: 554-563.

Nagaosa Y, Lanyon P, Doherty M(2002) Characterisation of size and direction of osteophyte in knee osteoarthritis: a radiographic study. Ann Rheum Dis 61: 319-324.

Nagel-Heyer S (2004) Tierversuchspilotstudie. In: Ingenieurtechnische Aspekte bei der Herstellung von dreidimensionalen Knorpel-Träger-Konstrukten (Engl.: Animal experiment pilot study. In: Engineering Aspects in Producing Three Dimensional Cartilage-CarrierConstructs), PhD Thesis, Books on Demand, Norderstedt, pp 89-107.

Nagel-Heyer S, Goepfert C, Feyerabend F, Petersen JP, Adamietz P, Meenen NM, Pörtner R (2005) Bioreactor cultivation of three-dimensional cartilage-carrierconstructs. Bioprocess Biosyst Eng 27: 273-280.

Nissi MJ, Rieppo J, Toyras J, Laasanen MS, Kiviranta I, Jurvelin JS, Nieminen MT (2006) T(2) relaxation time mapping reveals age- and species-related diversity of collagen network architecture in articular cartilage. Osteoarthritis Cartilage 14: 1265-1271.

Pauwels F, Maquet P, Furlong R (1980) The structure of the superficial layer of the articular cartilage of the glenoid cavity as an example of an embodied stress field. In: Biomechanics of the Locomotor Apparatus : Contributions on the Functional Anatomy of the Locomotor Apparatus (F. Pauwels, P. Maquet, R. Furlong, eds), Springer, Berlin, pp 329-374.
Petersen JP, Ueblacker P, Goepfert C, Adamietz P, Baumbach K, Stork A, Rueger JM, Poertner R, Amling M, Meenen NM (2008) Long term results after implantation of tissue engineered cartilage for the treatment of osteochondral lesions in a minipig model. J Mater Sci Mater Med 19: 2029-2038.

Poole CA, Flint MH, Beaumont BW (1984) Morphological and functional interrelationships of articular cartilage matrices. J Anat 138: 113-138.

Poole CA, Flint MH, Beaumont BW (1987) Chondrons in cartilage: ultrastructural analysis of the pericellular microenvironment in adult human articular cartilages. J Orthop Res 5: 509-522.

Rieppo J, Hallikainen J, Jurvelin JS, Kiviranta I, Helminen HJ, Hyttinen MM (2008) Practical considerations in the use of polarized light microscopy in the analysis of the collagen network in articular cartilage. Microsc Res Tech 71: 279-287.

Rieppo J, Hyttinen MM, Halmesmaki E, Ruotsalainen H, Vasara A, Kiviranta I, Jurvelin JS, Helminen HJ (2009) Changes in spatial collagen content and collagen network architecture in porcine articular cartilage during growth and maturation. Osteoarthritis Cartilage 17: 448-455.

Roberts S, Menage J, Sandell LJ, Evans EH, Richardson JB (2009) Immunohistochemical study of collagen types I and II and procollagen IIA in human cartilage repair tissue following autologous chondrocyte implantation. Knee 16: 398-404.

Seidel P, Hanke G, Grunder W (2005) Load distribution of articular cartilage from MR-images by neural nets. Z Med Phys 15: 101-106.

Setton LA, Elliott DM, Mow VC (1999) Altered mechanics of cartilage with osteoarthritis: human osteoarthritis and an experimental model of joint degeneration. Osteoarthritis Cartilage 7: 2-14.

van der Esch M, Steultjens M, Wieringa H, Dinant H, Dekker J (2005) Structural joint changes, malalignment, and laxity in osteoarthritis of the knee. Scand J Rheumatol 34: 298-301.

Xia Y, Moody JB, Alhadlaq H, Hu J (2003) Imaging the physical and morphological properties of a multi-zone young articular cartilage at microscopic resolution. J Magn Reson Imaging 17: 365-374.

Yaeger PC, Masi TL, de Ortiz JL, Binette F, Tubo R, McPherson JM (1997) Synergistic action of transforming growth factor-beta and insulin-like growth factor-I induces expression of type II collagen and aggrecan genes in adult human articular chondrocytes. Exp Cell Res 237: 318-325.

\section{Discussion with Reviewers}

Reviewer I: The differences in collagen distribution after 52 weeks are quite striking. Is it possible that the implanted engineered tissue is performing the function of 'filling the void' and providing conditions so that the native chondrocytes in the superficial zone can come in from the edges and make an attempt at regenerating the tissue? Authors: Unfortunately, we were not able to find any evidence for this interesting suggestion in our data. Although the tissue sections were made over the whole 
area of the implanted tissue, the data shown in this paper concentrate on the central portions of the tissue. The border between the implanted and the surrounding tissue is still clearly visible in nearly all of the samples, even after 52 weeks. At the interface between the implant and the native tissue, there was some cell death (empty lacunae) and also clustering of chondrocytes occurring in the vicinity of the incision made in the tissues during implantation. Particularly in the native cartilage directly surrounding the incision, there was a reduced cell density compared to the tissue further away from the implant site. Thus, neither in the surrounding cartilage tissue nor in the implant were there higher cell densities suggesting progenitor cells at the cartilage surface that proliferate and migrate from the edges towards the centre of the implant.

Reviewer II: What comment do the authors have about sample E with respect to its reduced depth and relatively poor morphology (compared to the other implants and native tissue)? Do you think this implant may have failed?
Authors: As shown for specimen E in Fig. 5 and Fig. 8, the height of the tissue overlying the subchondral bone is reduced at 26 weeks, even in comparison with the implants after 52 weeks. Furthermore, the anisotropic collagen fibre network is oriented predominantly parallel to the surface (specimen E in Fig. 5) and staining for collagen type I is more intense compared to collagen type II. Although collagen staining is not quantitative, this finding suggests a fibrous phenotype. This is in contrast to sample $\mathrm{D}$, where collagen type II staining was more prominent compared to collagen type I. Interestingly, the subchondral bone was closed in sample E, whereas in sample D the process of bone regeneration was not yet terminated. While the cartilage to bone interface of sample D was at the same level as within the surrounding tissue, the boundary in sample E was advanced towards the cartilage zone. Inappropriate load distribution may have contributed to the poor outcome of sample E, either as a cause for the negative development of the tissue or at least as a negative influence on tissue development. 